Portland State University

PDXScholar

$10-1-2012$

\title{
Decay rates of a molecule in the vicinity of a spherical surface of an isotropic magnetodielectric material
}

H. Y. Chung

P.T. Leung

Portland State University

D. P. Tsai

Follow this and additional works at: https://pdxscholar.library.pdx.edu/phy_fac

Part of the Physics Commons

Let us know how access to this document benefits you.

Citation Details

Chung, H. Y., Leung, P. T., Tsai, D. P. (2012). Decay rates of a molecule in the vicinity of a spherical surface of an isotropic magnetodielectric material. Physical Review B, 86(15), 155413.

This Article is brought to you for free and open access. It has been accepted for inclusion in Physics Faculty Publications and Presentations by an authorized administrator of PDXScholar. Please contact us if we can make this document more accessible: pdxscholar@pdx.edu. 


\title{
Decay rates of a molecule in the vicinity of a spherical surface of an isotropic magnetodielectric material
}

\author{
H. Y. Chung (鍾弘毅), ${ }^{1}$ P. T. Leung (梁培德) ${ }^{1,2,3}$ and D. P. Tsai (蔡定平 $)^{1,2}$ \\ ${ }^{1}$ Research Center for Applied Sciences, Academia Sinica, Taipei 115, Taiwan, Republic of China \\ ${ }^{2}$ Department of Physics, National Taiwan University, Taipei 10617, Taiwan, Republic of China \\ ${ }^{3}$ Department of Physics, Portland State University, P. O. Box 751, Oregon 97207-0751, USA
}

(Received 22 August 2012; revised manuscript received 19 September 2012; published 9 October 2012)

\begin{abstract}
A comprehensive study is presented on the decay rates of excited molecules in the vicinity of a magnetodielectric material of spherical geometry via electrodynamic modeling. Both the models based on a driven-damped harmonic oscillator and on energy transfers will be applied so that the total decay rates can be rigorously decomposed into the radiative and the nonradiative rates. Clarifications of the equivalence of these two models for arbitrary geometry will be provided. Different possible orientations and locations of the molecule are studied with the molecule being placed near a spherical particle or a cavity. Among other results, TE modes are observed which can be manifested via nonradiative transfer from a tangential dipole within a small range of dissipation parameters set for the spherical particle. In addition, spectral analysis shows that decay rates at such a particle with small absorption are largely dominated by radiative transfer except at multipolar resonances when nonradiative transfer becomes prominent, and relatively unmodified decay is possible when negative refraction takes place.
\end{abstract}

DOI: $10.1103 /$ PhysRevB.86.155413

PACS number(s): 33.70.Ca, 78.20.Bh, 78.67.Pt, 78.68.+m

\section{INTRODUCTION}

The problem of modified decay rates for molecules in the vicinity of a material body has been studied over several decades since the pioneering work of Purcell. ${ }^{1}$ It has been well established that such modification depends crucially on both the geometry and the optical properties of the material body (the "environment"). For a given molecule-environment configuration, the electromagnetic interaction between them will lead to certain modification of the photonic mode density and hence the molecular transition rate according to Fermi's golden rule. ${ }^{2}$ Over the years, this phenomenon has been studied with all kinds of materials including metals and nonmetals as well as different kinds of geometry such as planar, ${ }^{2,3}$ spherical, ${ }^{4-11}$ and roughened surfaces. ${ }^{12}$ In addition, the electromagnetic interaction as well as the molecule-environment system involved in the problem have been studied using different approaches including nonlocal optics ${ }^{13,14}$ as well as ${\text { both } \text { classical }^{3} \text { and quantum mechanical descriptions. }}^{2,15,16}$

The recent realization of negative refractive metamaterials whose possibility was first predicted in $1968^{17}$ has stimulated immense activity in the study of various optical processes in the presence of these new materials. ${ }^{18}$ In particular, the Purcell effect described above which is relevant to the emission properties of the molecules has also been studied for both planar $^{19-21}$ and spherical ${ }^{22-24}$ geometry, with unique modifications discovered from the interaction with such materials including anomalous blueshifts in the emission frequency, ${ }^{25}$ as well as the possibility for suppressed, ${ }^{20}$ enhanced, ${ }^{21-23,25}$ and relatively unmodified ${ }^{19,24}$ decay rates. With the advent of nanoparticle technology, the understanding of these effects in the vicinity of spherical particles is of prominent significance. However, to our knowledge, such modified molecular decay rates near a negative-refracting spherical particle have been studied only rarely in the literature, ${ }^{22,23}$ and even in these works the dissipation of the particle has been ignored. As for the study of the molecular decay due to the interaction with a dissipative metamaterial, we are aware of a few previous works which were limited to planar geometry, ${ }^{21,25}$ While the work of Moroz ${ }^{11}$ had indeed considered dissipative magnetodielectric spheres in its formalism, the magnetic effects were not studied in details and the relation between the total rate and the radiative and nonradiative rates was not elaborated in the general dissipative case. In a very recent work of Guzatov and Klimov, ${ }^{26}$ the authors had studied the more general case of an anisotropic and dissipative metamaterical sphere, but again, the decomposition of the total rate into the radiative and nonradiative rates was not considered. In an earlier work of Dung et al., ${ }^{24}$ which studied the decay of a molecule inside a spherical cavity of negative refracting materials, dissipation is considered but numerical studies were limited to the case with the molecule located at the center of the cavity.

The study of decay rates for a molecule-sphere system has a long history starting from the works of Gersten and Nitzan in the early $1980 \mathrm{~s}^{4-10}$ Recent experiments with metallic nanoparticles have studied this phenomenon down to very close distances between the molecule and the particle. ${ }^{27,28}$ One of the simple descriptions of the phenomenon is to model the decaying molecule as a damped harmonic oscillator driven by the fields reflected from the particle. ${ }^{3}$ Under the assumption that the natural and modified linewidths are small compared to the emission frequency, the modified normalized total decay rate can be obtained to be essentially the same as that from a more rigorous quantum linear response theory. ${ }^{24,29}$ In another approach from energy-transfer considerations, ${ }^{3,5,30}$ the modified decay rates can be separated into each of the radiative and nonradiative rates with the latter referring to the energy transferred from the dipole and absorbed by the sphere. ${ }^{4-7,14}$ While the two approaches have been available in the literature for some time, the equivalence between them was rigorously clarified only recently via an explicit electrodynamic calculation of various rates for the dipolesphere problem. ${ }^{31}$

It is the purpose of our present work to provide a general phenomenological theory via electrodynamic modeling for 
the treatment of modified decay rates for a molecule in the vicinity of a spherical surface of negative refracting magnetodielectric metamaterial. This theory will allow for (1) arbitrary locations for the molecule (both inside and outside the spherical surface), (2) materials with dissipation, and (3) rigorous separation into radiative and nonradiative rates when the molecule is outside the spherical particle. We shall employ both the mechanical (damped-oscillator) and the energy-transfer models and shall provide a clarification of their equivalence (valid for magnetodielectric material of arbitrary geometry) following the work of Barnett et al., ${ }^{29}$ without resorting to explicit calculations as was done in most previous works. ${ }^{6,11,31}$ Our work thus generalizes the previous work of Klimov ${ }^{22,23}$ to dissipative metamaterials with the calculated decay rates rigorously separated into radiative and nonradiative components, and extends that of Dung et al. ${ }^{24}$ to the study of molecules at arbitrary positions in the cavity so that the effect due to different molecular orientations can be studied. For simplicity, we shall limit ourselves to linear and isotropic magnetodielectric materials in the following formalism. ${ }^{17,19-24,32}$

\section{DECOMPOSITION OF DECAY RATES}

By modeling the molecule with a dipole moment $p_{0}$ as a damped oscillator of frequency $\omega_{0}$ driven by the reflected field $E_{R}$ from the environment, the overall decay rate (normalized to the intrinsic rate $\gamma_{0}$ ) in the presence of the environment can be obtained in the following form (under the assumption that both $\left.\gamma_{0}, \gamma \ll \omega_{0}\right):^{3}$

$$
\frac{\gamma}{\gamma_{0}}=1+\frac{3}{2 k^{3}} \operatorname{Im}\left(\frac{E_{R}}{p_{0}}\right)
$$

where we have assumed that the molecule is located in vacuum and $k$ is the wave number of the molecular emission. Note that we have also assumed the ideal case with the intrinsic quantum yield being unity and shall further simplify the calculation by evaluating $E_{R}$ at the unshifted frequency $\omega_{0} \cdot{ }^{3,33}$ It is well known that the result in Eq. (1) can also be justified from a more rigorous quantum mechanical approach by either solving the two-level Schrodinger equation in the second quantized formulation, ${ }^{24}$ or by resorting to the fluctuation-dissipation theorem. ${ }^{29}$

In another approach within this phenomenological modeling using classical electrodynamics, the total decay rate in Eq. (1) can be calculated in a different way by considering the energy transfer rate from the excited molecule to the environment via radiation (to infinity) and dissipation (absorption) by the materials of the environment. One thus obtains in this approach the following well-known expressions for the normalized radiative and nonradiative decay rates for the molecule:

$$
\frac{\gamma^{R}}{\gamma_{0}}=\frac{3}{c k^{4} p_{0}^{2}} \int_{(r \rightarrow \infty)} d \Omega r^{2} \vec{S} \cdot \hat{n}
$$

and

$$
\frac{\gamma^{N R}}{\gamma_{0}}=\frac{3}{c k^{4} p_{0}^{2}}\left(\frac{\omega}{8 \pi} \int \mu^{\prime \prime} \vec{H}_{0} \cdot \vec{H}_{0}^{*} d V+\frac{1}{2} \int \sigma \vec{E}_{0} \cdot \vec{E}_{0}^{*} d V\right),
$$

where $\vec{S}=\frac{c}{8 \pi} \operatorname{Re}\left(\vec{E} \times \vec{H}^{*}\right)$ is the time-averaged Poynting vector, $\omega$ is the emission frequency, and the second term in Eq. (3) is the well-known nonradiative rate due to joule heating of the particle ${ }^{3-5,7}$ where the conductivity is related to the imaginary part of the dielectric function via $\sigma=\frac{\omega \varepsilon^{\prime \prime}}{4 \pi}$. The first term in Eq. (3) is originated from the magnetic loss of the environment material, with $\mu^{\prime \prime}$ being the imaginary part of the permeability, ${ }^{11}$ and is often ignored in most previous works $^{3-5,7}$ in which only nonmagnetic materials were considered. Note that although we have quoted expressions in Gaussian units, the results in Eqs. (1)-(3) are universal and have the same values in any unit system since they are normalized rates.

While the results in Eqs. (1)-(3) are all well known, the exact decomposition of Eq. (1) into the rates in Eqs. (2) and (3) is a subtle issue and is not always clearly elaborated in the literature. This issue, namely, the equivalence between the mechanical (oscillator) model and the energy-transfer model, has been either taken for granted $^{4,7-9}$ or sometimes justified merely via explicit tedious calculations of the various rates subjected to certain specific configurations such as planar ${ }^{3}$ and spherical ${ }^{6,11,31}$ geometries. In the literature, we are aware of only the work by Barnett et al. ${ }^{29}$ which had presented a clear justification of the decomposition of the total rate in Eq. (1) into the rates in Eqs. (2) and (3). Since the work by Barnett et ll. $^{29}$ was formulated in second-quantization language and was limited to nonmagnetic materials, we have provided in the Appendix a classical electrodynamic decomposition of such rates including the magnetic loss rate as displayed in the first term of Eq. (3). In the following, we shall assume such decomposition is valid and apply it to the calculation of the various decay rates for a molecule in the vicinity of a magnetodielectric spherical surface.

\section{CALCULATION OF DECAY RATES}

The calculation of decay rates for molecular dipoles in the vicinity of a spherical surface with the dipole both outside and inside the surface has a long history. ${ }^{4-11}$ In this section, we shall provide details only for the new rates due to magnetic loss as described in Eq. (3), and shall simply collect the other results available in the literature with the Mie coefficients to depend also on the permeabilities. ${ }^{6}$

\section{A. Case for a spherical particle}

Consider an emitting dipole in vacuum interacting with a sphere of radius $a$ and material with linear and dissipative magnetodielectric properties as described above. To calculate the various decay rates, one simply follows the procedures in Ref. 5 to obtain the fields both inside and outside the sphere and obtain similar results for the total, radiative, and the joule loss nonradiative rates as follows (where all $\gamma$ 's below represent normalized rates). $5,7,11,31$

(i) For dipoles with radial orientation,

$$
\begin{aligned}
& \gamma_{\perp}=1+\frac{3}{2} \operatorname{Re} \sum_{n=1}^{\infty}(2 n+1) n(n+1) b_{n}\left[h_{n}(\rho) / \rho\right]^{2}, \\
& \gamma_{\perp}^{R}=\frac{3}{2} \sum_{n=1}^{\infty}(2 n+1) n(n+1) \frac{\left|j_{n}(\rho)+b_{n} h_{n}(\rho)\right|^{2}}{\rho^{2}},
\end{aligned}
$$




$$
\begin{aligned}
\gamma_{\perp}^{N R}= & \frac{3 x \varepsilon^{\prime \prime}}{2 \rho^{2}\left(\mu^{\prime} \varepsilon^{\prime \prime}+\mu^{\prime \prime} \varepsilon^{\prime}\right)} \sum_{n=1}^{\infty} n(n+1)\left|\beta_{n} h_{n}(\rho)\right|^{2} \\
& \times\left[(n+1) I_{n-1}+n I_{n+1}\right],
\end{aligned}
$$

where $k=\omega / c$ is the emission wave number, $x=k a, x_{1}=$ $\sqrt{\varepsilon \mu} k a, \rho=k r, j_{n}$ and $h_{n}$ are the spherical Bessel and Hankel functions of the first kind, respectively, and $I_{n}=$ $\operatorname{Im}\left[x_{1}^{*} j_{n-1}^{*}\left(x_{1}\right) j_{n}\left(x_{1}\right)\right] .^{31}$

(ii) For dipoles with tangential orientation,

$$
\gamma_{\|}=1+\frac{3}{4} \operatorname{Re} \sum_{n=1}^{\infty}(2 n+1)\left\{a_{n} h_{n}^{2}(\rho)+b_{n}\left(\frac{\left[\rho h_{n}(\rho)\right]^{\prime}}{\rho}\right)^{2}\right\},
$$

$$
\begin{gathered}
\gamma_{\|}^{R}=\frac{3}{4} \sum_{n=1}^{\infty}(2 n+1)\left\{\left|j_{n}(\rho)+a_{n} h_{n}(\rho)\right|^{2}\right. \\
\left.+\frac{1}{\rho^{2}}\left|\left[\rho j_{n}(\rho)\right]^{\prime}+b_{n}\left[\rho h_{n}(\rho)\right]^{\prime}\right|^{2}\right\}, \\
\gamma_{\|}^{N R}=\frac{3 x \varepsilon^{\prime \prime}}{4\left(\mu^{\prime} \varepsilon^{\prime \prime}+\mu^{\prime \prime} \varepsilon^{\prime}\right)} \sum_{n=1}^{\infty} A_{n},
\end{gathered}
$$

where

$$
\begin{aligned}
A_{n}= & \left|\alpha_{n} h_{n}(\rho)\right|^{2}(2 n+1) I_{n}+\left|\frac{\beta_{n}\left[\rho h_{n}(\rho)\right]^{\prime}}{\rho}\right|^{2} \\
& \times\left[(n+1) I_{n-1}+n I_{n+1}\right] .
\end{aligned}
$$

The Mie coefficients for magnetodielectric materials are given as $6,22,23$

$$
\begin{aligned}
a_{n} & =\frac{\mu\left[x j_{n}(x)\right]^{\prime} j_{n}\left(x_{1}\right)-\left[x_{1} j_{n}\left(x_{1}\right)\right]^{\prime} j_{n}(x)}{\left[x_{1} j_{n}\left(x_{1}\right)\right]^{\prime} h_{n}(x)-\mu\left[x h_{n}(x)\right]^{\prime} j_{n}\left(x_{1}\right)}, \\
b_{n} & =\frac{\varepsilon\left[x j_{n}(x)\right]^{\prime} j_{n}\left(x_{1}\right)-\left[x_{1} j_{n}\left(x_{1}\right)\right]^{\prime} j_{n}(x)}{\left[x_{1} j_{n}\left(x_{1}\right)\right]^{\prime} h_{n}(x)-\varepsilon\left[x h_{n}(x)\right]^{\prime} j_{n}\left(x_{1}\right)}, \\
\alpha_{n} & =\mu \frac{j_{n}(x)\left[x h_{n}(x)\right]^{\prime}-h_{n}(x)\left[x j_{n}(x)\right]^{\prime}}{\mu j_{n}\left(x_{1}\right)\left[x h_{n}(x)\right]^{\prime}-h_{n}(x)\left[x_{1} j_{n}\left(x_{1}\right)\right]^{\prime}}, \\
\beta_{n} & =\sqrt{\varepsilon \mu} \frac{j_{n}(x)\left[x h_{n}(x)\right]^{\prime}-h_{n}(x)\left[x j_{n}(x)\right]^{\prime}}{\varepsilon j_{n}\left(x_{1}\right)\left[x h_{n}(x)\right]^{\prime}-h_{n}(x)\left[x_{1} j_{n}\left(x_{1}\right)\right]^{\prime}} .
\end{aligned}
$$

In order to study the contribution of the magnetic loss to the nonradiative energy transfer we have to find the magnetic field inside the particle. Using the spherical vector wave functions $\vec{M}_{v}$ and $\vec{N}_{v}$ the electric field inside the sphere can be expressed $\mathrm{as}^{5}$

$$
\vec{E}(\vec{r}, \omega)=\sum_{v}\left(f_{v} \vec{M}_{v}^{1}\left(k_{1} r\right)+g_{v} \vec{N}_{v}^{1}\left(k_{1} r\right)\right),
$$

where $k_{1}=\sqrt{\varepsilon \mu} \omega / c$ is the wave vector inside the sphere and $f_{v}, g_{v}$, and the index $v$ are as defined in Ref. 5. Applying the relations

$$
\vec{\nabla} \times \vec{M}_{v}\left(k_{1} r\right)=k_{1} \vec{N}_{v}\left(k_{1} r\right), \quad \vec{\nabla} \times \vec{N}_{v}\left(k_{1} r\right)=k_{1} \vec{M}_{v}\left(k_{1} r\right),
$$

the magnetic field can be derived:

$$
\begin{aligned}
\vec{H}(\vec{r}, \omega) & =-(i c / \omega \mu) \vec{\nabla} \times \vec{E} \\
& =-i \sqrt{\frac{\varepsilon}{\mu}} \sum_{\nu}\left(f_{v} \vec{N}_{v}^{1}\left(k_{1} r\right)+g_{v} \vec{M}_{v}^{1}\left(k_{1} r\right)\right) .
\end{aligned}
$$

Next, substituting $\vec{H}$ into the first term in Eq. (3) leads to (with $\gamma_{M}^{N R} \equiv \gamma^{N R} / \gamma_{0}$ for this term)

$$
\begin{aligned}
\gamma_{M}^{N R}= & \frac{3}{c k^{4} p_{0}^{2}} \frac{\omega}{8 \pi} \int \mu^{\prime \prime} \vec{H}_{0} \cdot \vec{H}_{0}^{*} d V \\
= & \frac{3 \mu^{\prime \prime}}{8 \pi k^{3} p_{0}^{2}} \sum_{\nu} e_{m} \frac{(n-m) !}{n(n+1)(n+m) !} \\
& \times\left\{\left|f_{v}\right|^{2} \int_{0}^{a}\left[(n+1)\left|j_{n-1}\left(k_{1} r\right)\right|^{2}+n\left|j_{n+1}\left(k_{1} r\right)\right|^{2}\right] r^{2} d r\right. \\
& \left.+\left|g_{v}\right|^{2}(2 n+1) \int_{0}^{a}\left|j_{n}\left(k_{1} r\right)\right|^{2} r^{2} d r\right\}
\end{aligned}
$$

where $e_{0}=1$ and $e_{m>0}=2$ as defined in Ref. 5 .

For the two special cases of radial and tangential dipoles, the result in Eq. (15) can be simplified using the explicit results of $f_{v}$ and $g_{v}$ available in Ref. 5. Together with the results in Eqs. (6) and (9), the final results for the total nonradiative rates in Eq. (3) can be obtained in the form

$$
\begin{aligned}
\gamma_{\perp}^{N R}= & \frac{3 x}{2 \rho^{2}\left(\mu^{\prime} \varepsilon^{\prime \prime}+\mu^{\prime \prime} \varepsilon^{\prime}\right)} \sum_{n=1}^{\infty} n(n+1)\left|\beta_{n} h_{n}(\rho)\right|^{2} \\
& \times\left\{\varepsilon^{\prime \prime}\left[(n+1) I_{n-1}+n I_{n+1}\right]+\frac{\mu^{\prime \prime}|\varepsilon|}{|\mu|}(2 n+1) I_{n}\right\}
\end{aligned}
$$

for radial dipoles and

$$
\gamma_{\|}^{N R}=\frac{3 x}{4\left(\mu^{\prime} \varepsilon^{\prime \prime}+\mu^{\prime \prime} \varepsilon^{\prime}\right)} \sum_{n=1}^{\infty}\left(\varepsilon^{\prime \prime} A_{n}+\frac{\mu^{\prime \prime}|\varepsilon|}{|\mu|} B_{n}\right)
$$

for tangential dipoles, where

$$
\begin{aligned}
B_{n}= & \left|\alpha_{n} h_{n}(\rho)\right|^{2}\left[(n+1) I_{n-1}+n I_{n+1}\right] \\
& +\left|\frac{\beta_{n}\left[\rho h_{n}(\rho)\right]^{\prime}}{\rho}\right|^{2}(2 n+1) I_{n} .
\end{aligned}
$$

Hence the complete results for the various decay rates for the dipole-magnetodielectric sphere problem are given in Eqs. (4), (5), (7), (8), (10), (11), and (16)-(18).

\section{B. Case for a spherical cavity}

Next we consider the complementary problem with the dipole in an arbitrary location inside a spherical cavity of vacuum surrounded by a linear dissipative magnetodielectric material extended to infinity. In this case one will have either purely nonradiative transfer, or purely radiative transfer when the dissipation of the material is ignored. ${ }^{7}$

It turns out that the results for this case can easily be obtained from the above results for a dipole-sphere system via a simple transformation based on reciprocity symmetry. ${ }^{7}$ Thus we propose that the cavity problem can simply be solved by using the results listed above and applying the following 

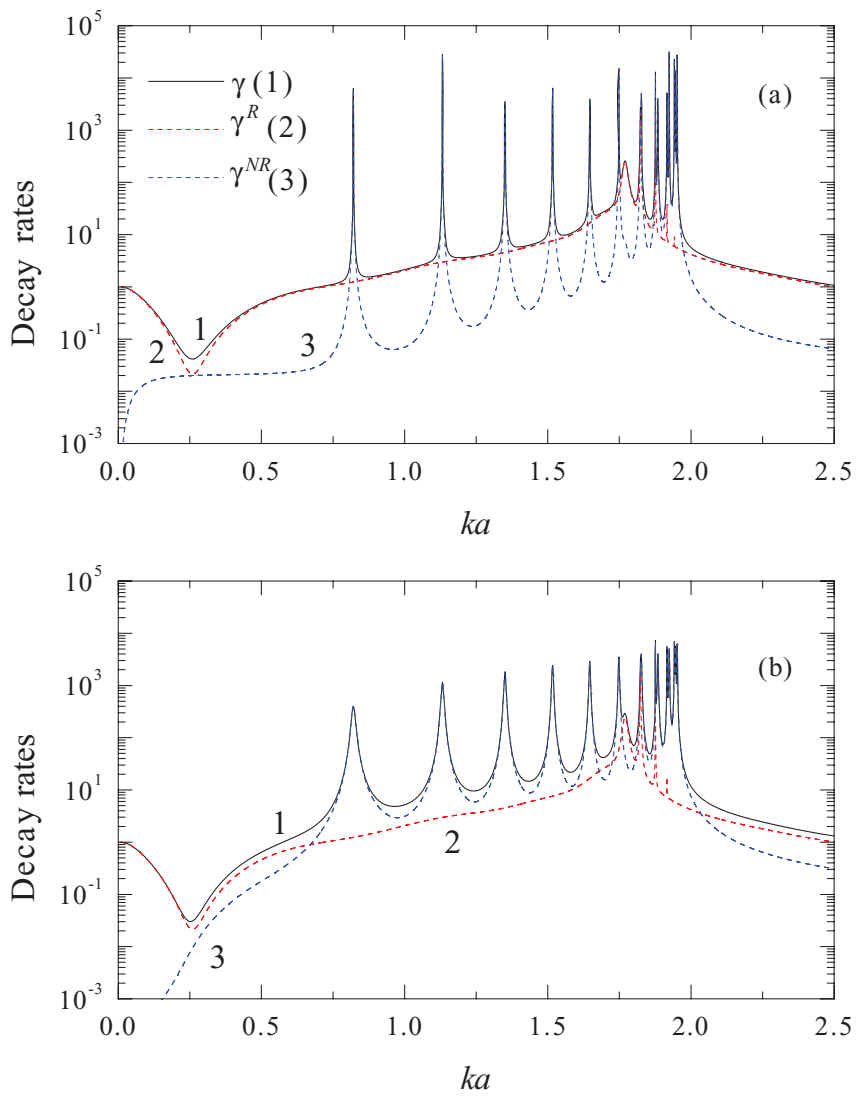

FIG. 1. (Color online) Total (curve 1), radiative (curve 2), and nonradiative (curve 3 ) decay rates of an oscillating dipole in the vicinity of a sphere with (a) $\varepsilon=-4+0.0005 i$ and $\mu=-1.05$ and (b) $\varepsilon=-4$ and $\mu=-1.05+0.00005 i$. The dipole is along the tangential direction and the gap between the dipole and the sphere is set to $k d=0.1$.

transformation rule:

$$
T\left[\begin{array}{c}
j_{n} \\
h_{n} \\
k_{1}, \varepsilon_{1}, \mu_{1} \\
k_{2}, \varepsilon_{2}, \mu_{2} \\
x_{1} \\
x_{2}
\end{array}\right]=\left[\begin{array}{c}
h_{n} \\
j_{n} \\
k_{2}, \varepsilon_{2}, \mu_{2} \\
k_{1}, \varepsilon_{1}, \mu_{1} \\
x_{2} \\
x_{1}
\end{array}\right] .
$$

The subscripts 1 and 2 in Eq. (19) denote quantities inside and outside the spherical boundary, respectively. Hence without worrying about the separation of the decay into radiative or nonradiative components in this case, we easily obtain the total decay rate by applying (19) to Eqs. (4) and (7) for the two dipole orientations in the cavity as follows: ${ }^{6,7}$

$$
\gamma_{\perp}=1+\frac{3}{2} \operatorname{Re} \sum_{n=1}^{\infty}(2 n+1) n(n+1) E_{n}\left[j_{n}(\rho) / \rho\right]^{2}
$$

and

$\gamma_{\|}=1+\frac{3}{4} \operatorname{Re} \sum_{n=1}^{\infty}(2 n+1)\left\{F_{n} j_{n}^{2}(\rho)+E_{n}\left(\frac{\left[\rho j_{n}(\rho)\right]^{\prime}}{\rho}\right)^{2}\right\}$,
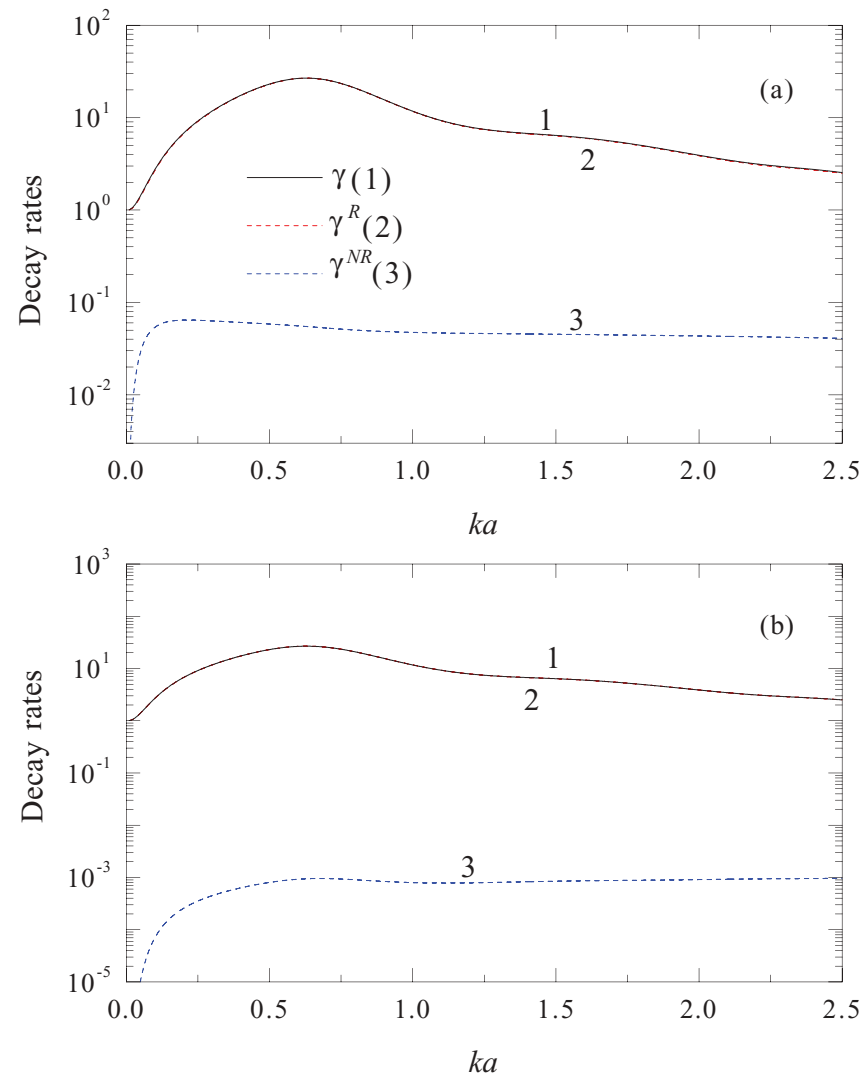

FIG. 2. (Color online) Same as in Fig. 1 but the dipole is along the radial direction.

where $E_{n}=T\left[b_{n}\right]$ and $F_{n}=T\left[a_{n}\right]$, respectively. As an example, for the special case when the dipole is located at the center of the cavity, one can easily check that either Eq. (20) or Eq. (21) will yield ${ }^{7} \gamma=1+\operatorname{Re}\left(E_{1}\right)$ which leads to the same result as that in Eq. (69) of Ref. 24. In the following, we shall allow the dipole to be at any location inside the cavity so that effects due to different molecular orientations will be revealed from the results in Eqs. (20) and (21).

\section{NUMERICAL RESULTS}

To illustrate the decay characteristics due to the absorption of the magnetodielectric particle, we start by referring back to the results of Klimov, ${ }^{22}$ which were limited to transparent (nonabsorptive) particles. In Klimov's work, which excludes any nonradiative transfer, it was demonstrated that for tangentially oriented (electric) dipoles, huge enhancement of decay rates can occur at the resonance of the so-called left-handed transverse electric (LHTE) surface modes when the sphere acquires a negative refractive index. In Fig. 1, we have recalculated the results using parameters as in Ref. 22 but with a small damping (imaginary part) added either to the permittivity or the permeability of the sphere (radius $a$ ). The results shown are for a tangential dipole with the decay separated into the radiative and nonradiative rates. We have located the dipole at a small distance from the particle surface $(k d=0.1)$ so that the nonradiative rate remains well-behaved. Figure 1(a) shows the results with a small imaginary part for $\varepsilon$, from which we see that while the radiative rate reproduces 


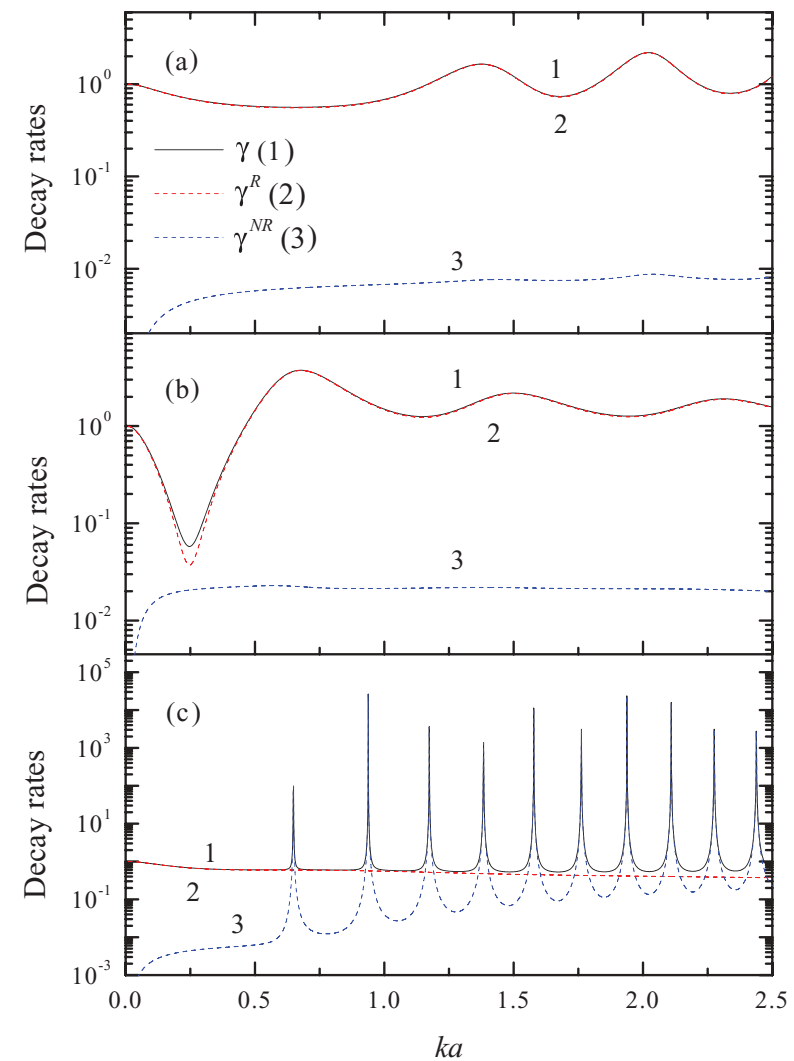

FIG. 3. (Color online) Same as in Fig. 1, but the permittivity and permeability of the sphere are (a) $\varepsilon=4+0.0005 i$ and $\mu=1.05$, (b) $\varepsilon=-4+0.0005 i$ and $\mu=1.05$, and (c) $\varepsilon=4+0.0005 i$ and $\mu=-1.05$.

essentially the same LHTE resonances (at $k a \sim 1.9$ ) as described in Ref. 22, there appears new extra resonances in the nonradiative rate which are due to the excitation of TE surface modes. ${ }^{19,22}$ Note that only $\mu<0$ but not LH behavior is required for these resonances (see the results below in Fig. 3), and they also emerge when a small imaginary part is instead added to $\mu$ as shown in Fig. 1(b). Note, however, the damping must be very small for such peaks to prevail and is even more restricted for the case with a complex permeability. In this latter case, we further notice that the broadening of the resonances is more severe compared to the case with a complex permittivity. In addition, such resonances are characteristics of surface TE modes of higher orders, and hence are not manifested in the radiative rates since it is well known that such modes are dominated by nonradiative transfers. ${ }^{35}$ We have further confirmed (not shown) the existence of these modes when both the permittivity and permeability are complex. For such small damping in Fig. 1, the whispering gallery mode (WGM) resonances ${ }^{22}$ at large $k a$ values prevail (not shown), but at greater damping they disappear together with the TE surface modes becoming severely broadened and almost disappear.

Figure 2 shows the corresponding results for a radial dipole with the same parameters as those in Fig. 1. Since such oriented dipole emits only TM waves, all the TE resonances observed in Fig. 1 disappear and the decay is largely radiative for such small damping constants. The WGM resonances at large $k a$
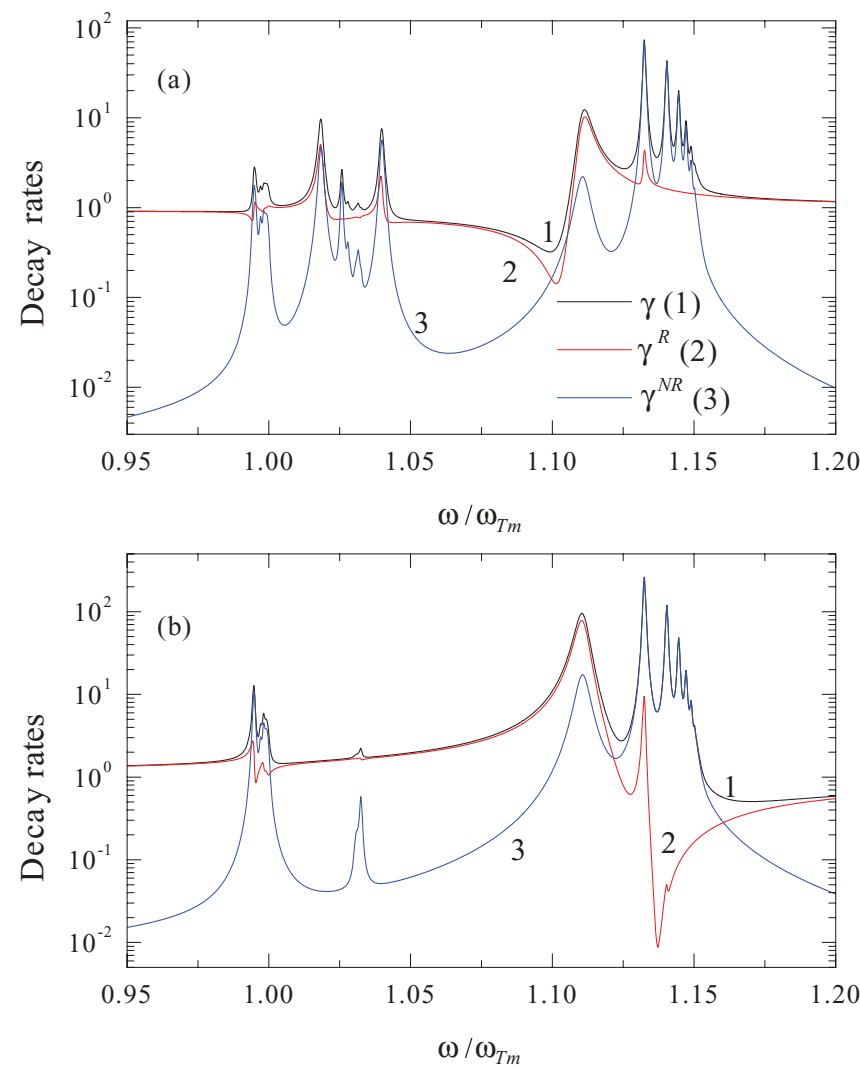

FIG. 4. (Color online) Decay rate spectrum for (a) a tangential dipole and (b) a radial dipole with the sphere radius and the moleculesphere separation set at $a=0.05 \lambda_{T m}$ and $d=0.05 \lambda_{m}$, respectively. Other parameters for the permittivity and permeability are set as in Ref. 25: $\omega_{T e}=1.03 \omega_{T m}, \omega_{P m}=0.43 \omega_{T m}$, and $\omega_{P e}=0.75 \omega_{T m}$, respectively.

values are again similar to those in Ref. 22 and are not shown here.

Figure 3 is plotted for three other special cases to help clarify the results observed in Fig. 1. In Figs. 3(a) and 3(b), the value of the permeability is set positive and a small damping is added to the permittivity with Fig. 3(a) being dielectriclike and Fig. 3(b) metal-like. In either case, all the TE resonances (LHTE and TE surface modes) disappear even for the case with a tangential dipole. In Fig. 3(c), however, the real part of the permittivity is set positive while the permeability is set negative. In this case we do not have negative refraction but the TE surface resonances are still seen as long as we have $\mu<0$. Moreover, the LHTE modes disappear as expected.

Next we study the spectral characteristics of the molecular decay in the presence of a magnetodielectric sphere. For this purpose, we shall adopt the same single-resonance Lorentz models for both the permittivity and permeability as used in Ref. 24. Thus we have

$$
\varepsilon(\omega)=1+\frac{\omega_{P e}^{2}}{\omega_{T e}^{2}-\omega^{2}-i \omega \Gamma_{e}}
$$

and

$$
\mu(\omega)=1+\frac{\omega_{P m}^{2}}{\omega_{T m}^{2}-\omega^{2}-i \omega \Gamma_{m}},
$$



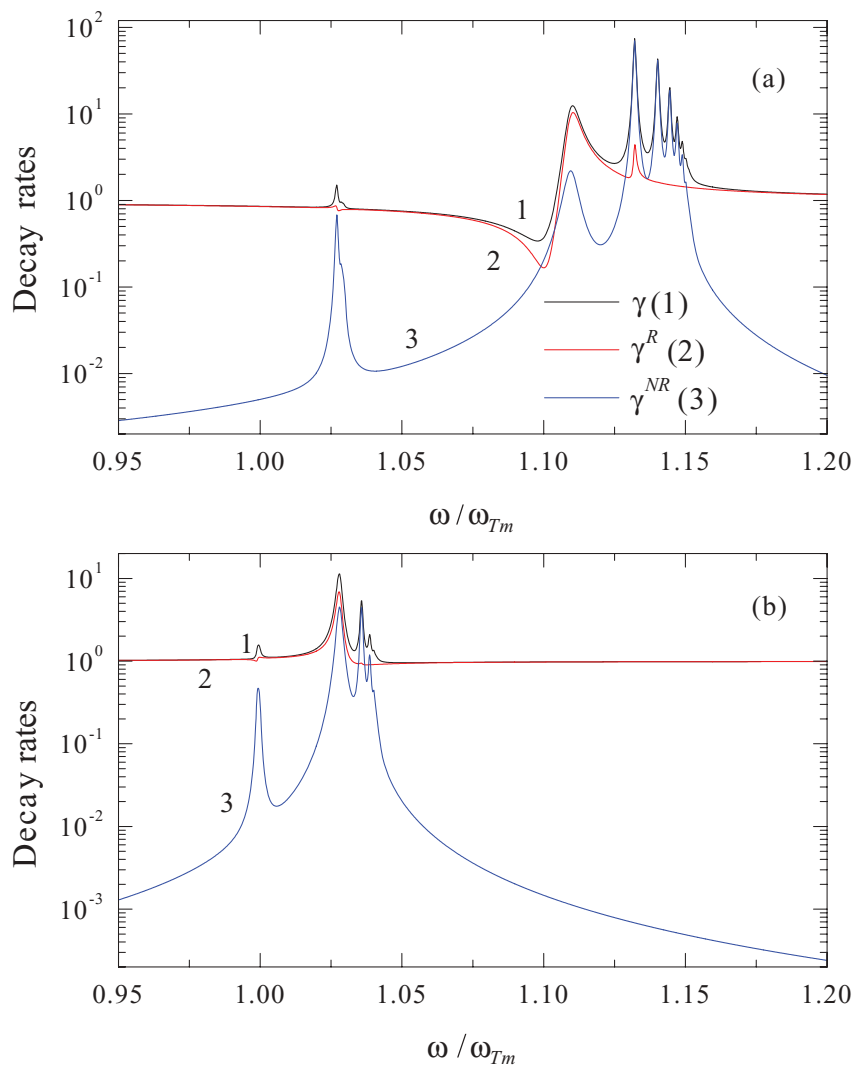

FIG. 5. (Color online) Similar to Fig. 4 for the tangential dipole for (a) a "pure electric" material $(\mu=1)$ and (b) a "pure magnetic" material $(\varepsilon=1)$.

where the values for the various parameters are as given in Ref. 24 and very small damping constants are used with $\Gamma_{e}=\Gamma_{m}=10^{-3} \omega_{T m}$. Following the analysis of Ref. 24, we have found the range of frequency for negative refraction to be around $1.017<\omega / \omega_{T m}<1.129$. Figure 4 shows the total decay rates for both a tangential and a radial dipole at a close distance $\left(d=0.05 \lambda_{T m}\right)$ from such a sphere of relatively small size $\left(a=0.05 \lambda_{T m}\right)$. It is seen that two groups of multipole resonance emerge (centered around $1.00 \omega / \omega_{T m}$ and $\left.1.15 \omega / \omega_{T m}\right)$, due each to the relative independent resonance of the electric [Eq. (22)] and magnetic [Eq. (23)] type. These can be confirmed by referring to Fig. 5 where each of the "pure electric $(\mu=1)$ " and "pure magnetic $(\varepsilon=1)$ " sphere is considered for the case with a tangential dipole (see similar illustrations in previous works). ${ }^{19,24,36}$ Note also from Fig. 4 that the resonance structure is simpler in the radial dipole case since only TM excitation is involved in this case. ${ }^{19}$ With the decomposition of the total rate into the radiative and nonradiative components, it is seen that for such small damping constants, the decay is mainly radiative in nature, except when being close to the resonances discussed above where the nonradiative transfer becomes dominant due to the multipolar characteristics of these modes. This is particularly significant at the high resonant frequencies of the electric type where the decay is largely nonradiative. Most interestingly, one observes that when negative refraction takes place, relative unmodified decay rates are seen due to the "almost transparency" of the sphere under this condition. ${ }^{19,24,36}$ We further show in Fig. 6 the
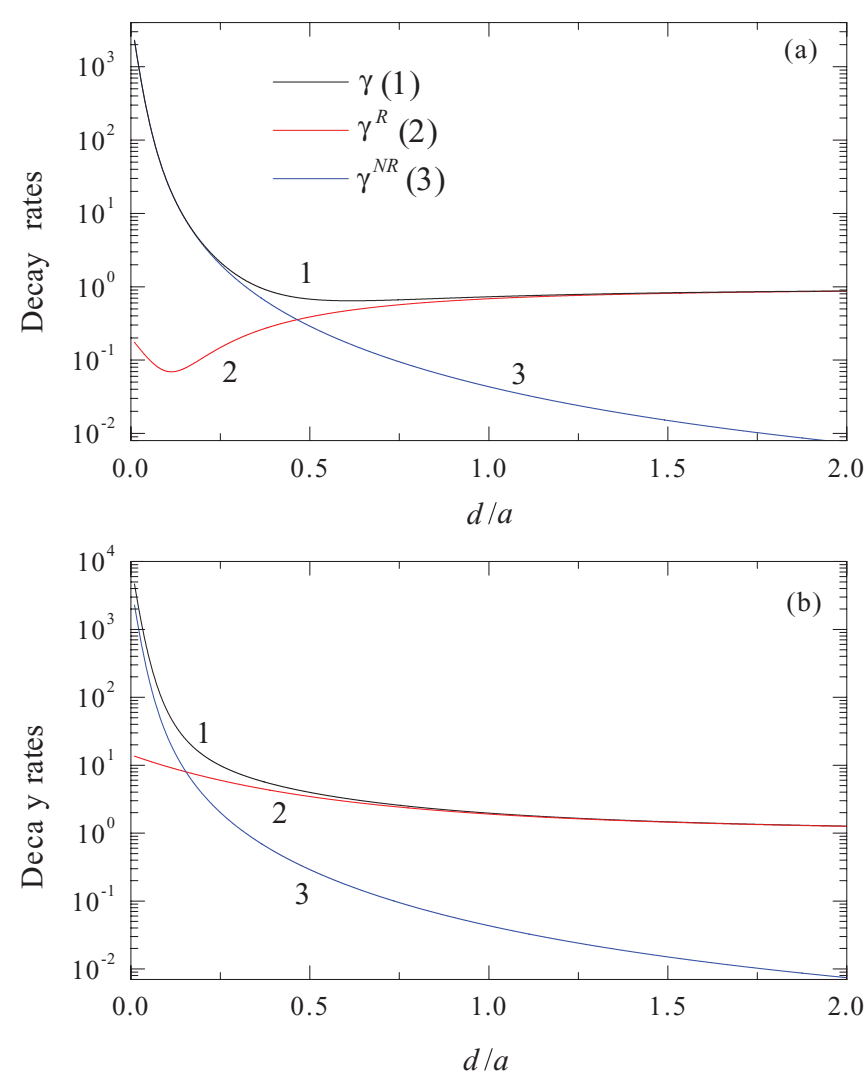

FIG. 6. (Color online) Distance dependence of the decay rates for the same problem as in Fig. 4 under the condition of negative refraction. The emission frequency is set at $\omega=1.05 \omega_{T m}$ at which we have the following values for the material constants: $\varepsilon=-12.51+$ $0.34 i, \mu=-0.803+0.018 i$, and $n=-3.17+0.08 i$.

distance dependence of the different rates under the condition of negative refraction and the behaviors are seen very similar to the case with ordinary metallic spheres with nonradiative transfer dominates at close distances for both tangential and radial dipoles, and the radiative rate for tangential dipoles can dip at close distances due to the opposite polarity of the induced dipole with respect to this orientation of the source dipole. $^{3}$

Finally, we study the decay problem with the molecule located in vacuum inside a spherical cavity of a magnetodielectric material of infinite extent as described by the properties in Eqs. (22) and (23). As explained in the above, we only need to examine the total decay rates using Eqs. (20) and (21) in this case. While Ref. 24 has studied this problem with the molecule located at the center of the cavity, we shall allow it to be at an arbitrary location so that orientation effects can be studied.

Using the model in Eqs. (22) and (23), we have plotted the decay rates for both oriented dipoles in Fig. 7 at positions both a little off the center [Fig. 7(b)] and very close to the cavity boundary [Fig. 7(c)], respectively. Figure 7(a) reproduces the result in Fig. 3(c) of Ref. 24 for reference. While it is seen that several higher order resonances $\left(1.1 \leqslant \omega / \omega_{T m} \leqslant\right.$ 1.2) of the "electric type" [from Eq. (22)] appear once the dipole is off the center (at $r=0.1 a$ ), these peaks fall into the TM and TE type with the latter excited only by the 


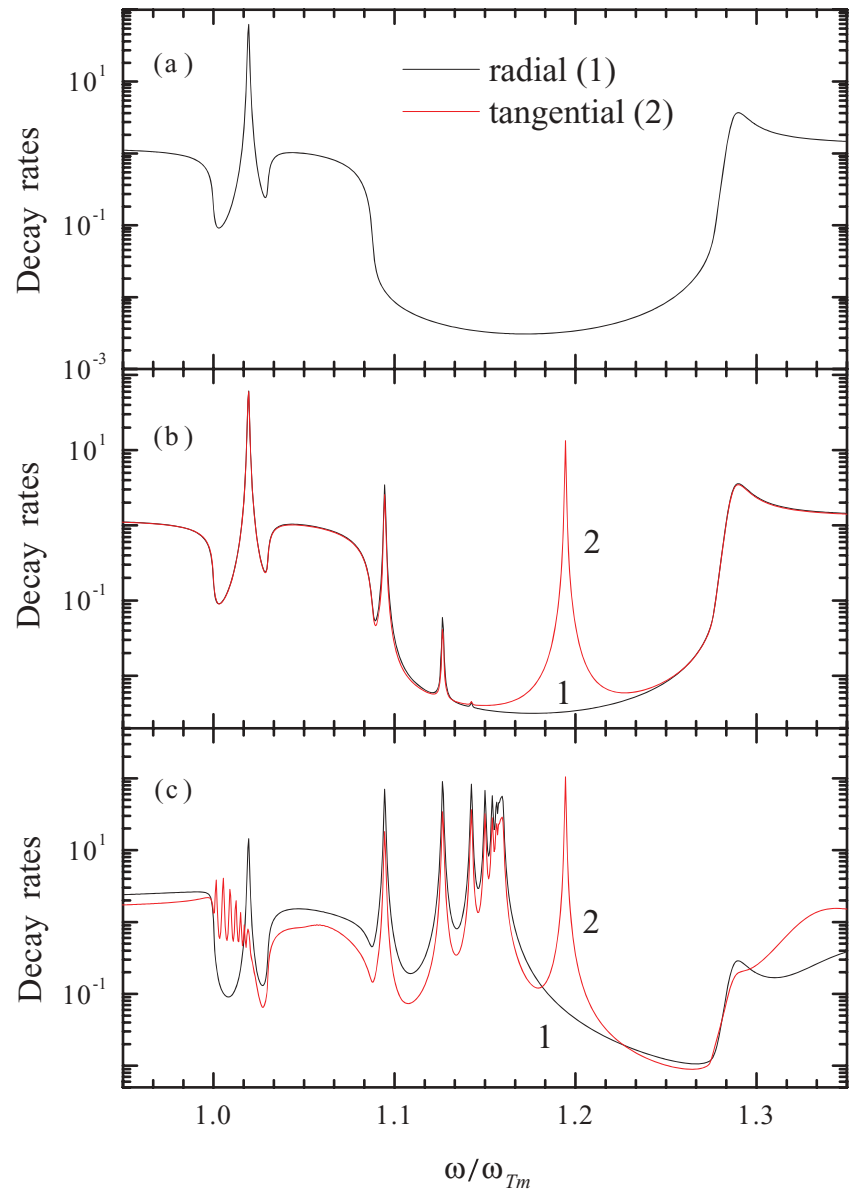

FIG. 7. (Color online) Decay rate spectrum for a dipole inside a vacuum cavity of material with $\varepsilon$ and $\mu$ as in Fig. 4. The dipole is along the radial (curve 1) or tangential (curve 2) direction and located at (a) $r=0$, (b) $r=0.1 a$, and (c) $r=0.8 a$. The radius of the cavity is fixed at $R=0.5 \lambda_{T m}$.

tangential dipole $\left(\omega / \omega_{T m} \sim 1.2\right)$. Note that, for cavity modes, higher order electric multipole resonances appear at lower frequencies. When the dipole is moved further away from the center to lie close to the cavity boundary [Fig. 7(c)], we see that more multipole resonances of the electric type appear. Most interestingly, extra magnetic resonances also appear $\left(\omega / \omega_{T m} \sim 1.0\right)$, which are coupled only to the tangential dipole. We have checked that these resonances occur under the conditions with $\operatorname{Re}(n)>0$ and $\operatorname{Re}(\mu)<0$, and they seem to have the same origin as the TE surface modes observed in Figs. 1 and 3 as described above. Again, the nearly unmodified decay rate is seen for negative refraction regime in all the results in Fig. $7 .^{24}$

\section{CONCLUSION}

In this work, we have studied using electrodynamic modeling the decay behavior of a dipole molecule in the vicinity of a spherical magnetodielectric surface. We start by following Barnett et al. ${ }^{29}$ to provide a rigorous justification of the decomposition of the total rate into the radiative and nonradiative components [Eqs. (1)-(3), and Appendix], which is valid for any geometry and media with linear response and can be both dispersive and dissipative. When these results are applied to the magnetodielectric materials, we observe TE resonances manifested in the nonradiative transfer between the molecule and the particle which were not seen previously when absorption was absent. ${ }^{22}$ In the event when negative refraction takes place, relatively unmodified decay rates are observed as reported previously in the literature. ${ }^{24}$ In the case of a spherical cavity surrounded by such materials of infinite extent, we have reproduced the previous results for the decay rates in the literature via a simple transformation ${ }^{7}$ and have applied them to the decay of molecules located off the cavity center. In this configuration, tangential and radial dipoles are distinguishable and we have observed resonance behavior in the decay of tangential dipoles analogous to the LHTE modes in the particle case. ${ }^{22}$

Our study thus complements the previous ones ${ }^{22,24}$ which together demonstrate the possibility to control the emission characteristics using particles or cavities made of magnetodielectric metamaterials. Still even richer possibilities may be available by exploring more complicated spherical structures made of these materials such as the multishell nanomatryoshka as in the case of plasmonic nanoshells. ${ }^{37}$ The previous formulation using transfer matrix by Moroz will be useful for the extension of the present study to these more complicated structures. ${ }^{11}$ Furthermore, our decomposition into the radiative and nonradiative rates are so far limited to isotropic magnetodielectric materials. It will be of interest to extend the present formulation (see Appendix) to decompose the result obtained by Guzatov and Klimov ${ }^{26}$ for the total decay rate at an anisotropic metamaterial particle, so that the separate contribution from radiative and nonradiative transfers can be studied in this more general case.

\section{ACKNOWLEDGMENTS}

This research is supported by financial aid from National Science Council, Taiwan under Grants No. NSC 100-2923M-002-007-MY3, No. NSC 101-3113-P-002-021, and No. NSC101-2112-M-002-023. We are also grateful to Molecular Imaging Center of National Taiwan University for their support.

\section{APPENDIX}

Here we provide a general proof of the decomposition of the total decay rate in Eq. (1) into the radiative and nonradiative rates in Eqs. (2) and (3), without resorting to explicit calculation of these rates for a specific configu-

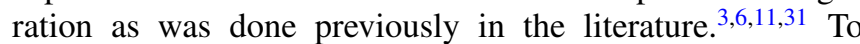
do this, we shall follow the method of Barnett et al. ${ }^{29}$ by resorting to the Poynting theorem. We begin by assuming the following constitutive relations: $\vec{B}=\mu \vec{H}$ and $\vec{D}=\varepsilon \vec{E}$ for the metamaterial environment with $\mu=\mu^{\prime}+i \mu$ and $\varepsilon=$ $\varepsilon^{\prime}+i \varepsilon^{\prime \prime}$, respectively, and that all the fields and source dipole are harmonic in time: $\vec{B}=\vec{B}_{0} e^{-i \omega t}, \vec{E}=\vec{E}_{0} e^{-i \omega t}$, and $\vec{p}=$ $\vec{p}_{0} e^{-i \omega t}$. Hence the current density and field corresponding to the point dipole can be expressed as $\vec{J}\left(\vec{r}^{\prime}\right)=-i \omega \vec{p} \delta\left(\vec{r}^{\prime}\right)$ 
and $\vec{E}(\vec{r})=\omega^{2} \overleftrightarrow{G}\left(\vec{r}^{\prime}, \vec{r}\right) \cdot \vec{p}\left(\vec{r}^{\prime}\right)$, respectively, where the Green dyadic is complex in general: $\stackrel{\leftrightarrow}{G}=\overleftrightarrow{G}^{\prime}+i \overleftrightarrow{G}^{\prime \prime}{ }^{34}$

From Maxwell's equations, one easily arrives at the following result:

$$
\vec{\nabla} \cdot(\vec{E} \times \vec{H})=-\frac{4 \pi}{c} \vec{J} \cdot \vec{E}-\frac{1}{c}\left(\vec{H} \cdot \frac{\partial \vec{B}}{\partial t}+\vec{E} \cdot \frac{\partial \vec{D}}{\partial t}\right) .
$$

Note that, although we adopt Gaussian units here since this was done in most previous works on this problem, $3,6,7,11,22,23,30,31$ the results obtained for the normalized rates in Eqs. (1)-(3) are universal and the same for all unit systems. Integrating over a volume which contains the dipole and the whole "environment" we obtain

$$
\begin{aligned}
\int d V \vec{\nabla} \cdot(\vec{E} \times \vec{H})= & \oint(\vec{E} \times \vec{H}) \cdot d \vec{a} \\
= & -\frac{4 \pi}{c} \int d V \vec{J} \cdot \vec{E}-\frac{1}{c} \int d V \\
& \times\left(\vec{H} \cdot \frac{\partial(\mu \vec{H})}{\partial t}+\vec{E} \cdot \frac{\partial(\varepsilon \vec{E})}{\partial t}\right) .
\end{aligned}
$$

Now applying the following theorem for time-averaging over products of harmonic fields (for both scalar and vector products):

$$
\langle\operatorname{Re} \vec{X} \otimes \operatorname{Re} \vec{Y}\rangle=\frac{1}{2} \operatorname{Re}\left(\vec{X} \otimes \vec{Y}^{*}\right)=\frac{1}{2} \operatorname{Re}\left(\vec{X}^{*} \otimes \vec{Y}\right),
$$

Eq. (A2) leads to

$$
\begin{aligned}
\oint & \operatorname{Re}\left(\vec{E} \times \vec{H}^{*}\right) \cdot d \vec{a} \\
= & -\frac{4 \pi}{c} \operatorname{Re} \int d V \vec{J} \cdot \vec{E}^{*}-\frac{1}{c} \operatorname{Re} \int d V\left(\vec{H} \cdot\left(i \omega \mu^{*} \vec{H}^{*}\right)\right. \\
& \left.+\vec{E} \cdot\left(i \omega \varepsilon^{*} \vec{E}^{*}\right)\right) .
\end{aligned}
$$

Simplification of the first term on the right-hand side (RHS) of Eq. (A4) leads to

$$
\begin{aligned}
\operatorname{Re} \int d V \vec{J} \cdot \vec{E}^{*}= & \operatorname{Re} \int d V\left(-i \omega \vec{p}_{0} e^{-i \omega t} \delta\left(\vec{r}_{p}\right)\right) \\
& \cdot \omega^{2}\left(\overleftrightarrow{G}^{\prime}-i \overleftrightarrow{G}^{\prime \prime}\right) \cdot \vec{p}_{0} e^{i \omega t} \\
= & -\omega^{3} \int d V \vec{p}_{0} \cdot \overleftrightarrow{G}^{\prime \prime} \cdot \vec{p}_{0} \delta\left(\vec{r}_{p}\right) \\
= & -\omega^{3} \vec{p}_{0} \cdot \operatorname{Im} \overleftrightarrow{G} \cdot \vec{p}_{0},
\end{aligned}
$$

where all the quantities in the last row are evaluated at the position of the dipole.
Next we consider the second term of the RHS of Eq. (A4):

$$
\begin{aligned}
& \operatorname{Re} \int d V\left(\vec{H} \cdot\left(i \omega \mu^{*} \vec{H}^{*}\right)+\vec{E} \cdot\left(i \omega \varepsilon^{*} \vec{E}^{*}\right)\right) \\
& =\operatorname{Re} \int d V\left(\left(\mu^{\prime}-i \mu^{\prime \prime}\right) \vec{H} \cdot\left(i \omega \vec{H}^{*}\right)+\left(\varepsilon^{\prime}-i \varepsilon^{\prime \prime}\right) \vec{E} \cdot\left(i \omega \vec{E}^{*}\right)\right) \\
& =\omega \int d V\left(\mu^{\prime \prime} \vec{H}_{0} \cdot \vec{H}_{0}^{*}+\varepsilon^{\prime \prime} \vec{E}_{0} \cdot \vec{E}_{0}^{*}\right) \\
& =\omega \int d V\left(\mu^{\prime \prime} \vec{H}_{0} \cdot \vec{H}_{0}^{*}\right)+4 \pi \int d V \sigma \vec{E}_{0} \cdot \vec{E}_{0}^{*},
\end{aligned}
$$

where we have used the following relation for the conductivity: $\sigma=\frac{\omega \varepsilon^{\prime \prime}}{4 \pi}$.

Substituting (A5) and (A6) into Eq. (A4), we finally obtain

$$
\begin{aligned}
\frac{c}{8 \pi} \oint \operatorname{Re}\left(\vec{E} \times \vec{H}^{*}\right) \cdot d \vec{a} \\
=\frac{\omega^{3}}{2} \vec{p}_{0} \cdot \operatorname{Im} \overleftrightarrow{G} \cdot \vec{p}_{0}-\frac{\omega}{8 \pi} \int \mu^{\prime \prime} \vec{H}_{0} \cdot \vec{H}_{0}^{*} d V \\
\quad-\frac{1}{2} \int \sigma \vec{E}_{0} \cdot \vec{E}_{0}^{*} d V .
\end{aligned}
$$

To convert the above result in Eq. (A7) to various normalized decay rates of the molecule in the presence of the environment, we divide through it by the time-averaged radiated power from a harmonic dipole: $W=\frac{1}{3} c k^{4} p_{0}^{2}$ and obtain the following result:

$$
\begin{aligned}
\frac{3 \omega^{3}}{2 c k^{4} p_{0}^{2}} \vec{p}_{0} \cdot \operatorname{Im} \overleftrightarrow{\leftrightarrow} \cdot \vec{p}_{0}= & \frac{3}{c k^{4} p_{0}^{2}} \frac{c}{8 \pi} \oint \operatorname{Re}\left(\vec{E} \times \vec{H}^{*}\right) \cdot d \vec{a} \\
& +\frac{3}{c k^{4} p_{0}^{2}}\left(\frac{\omega}{8 \pi} \int \mu^{\prime \prime} \vec{H}_{0} \cdot \vec{H}_{0}^{*} d V\right. \\
& \left.+\frac{1}{2} \int \sigma \vec{E}_{0} \cdot \vec{E}_{0}^{*} d V\right) .
\end{aligned}
$$

Now let us separate the Green dyadic on the LHS into the vacuum part and the scattering (reflection) part, $\stackrel{\leftrightarrow}{G}=$

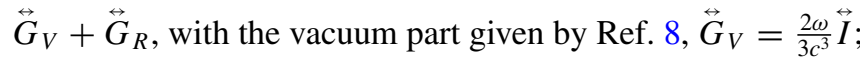
where $\overleftrightarrow{I}$ is the unit dyadic; then one sees that the LHS of Eq. (A8) leads to the normalized total decay rate in Eq. (1). Hence by introducing the normalized radiative and nonradiative rates defined as in Eqs. (2) and (3), we see that the result in Eq. (A8) provides a rigorous decomposition of the total decay rate into its radiative and nonradiative components, i.e., $\gamma=\gamma^{R}+\gamma^{N R}$. We remark that the above derivation is valid for a dipole interacting with an environment of linear material which can have arbitrary geometry, although we have only applied them to spherical geometry in our present work.
${ }^{1}$ E. M. Purcell, Phys. Rev. 69, 681 (1946).

${ }^{2}$ W. L. Barnes, J. Mod. Opt. 45, 661 (1998).

${ }^{3}$ R. R. Chance, A. Prock, and R. Silbey, Adv. Chem. Phys. 37, 1 (1978), and references therein.

${ }^{4}$ J. Gersten and A. Nitzan, J. Chem. Phys. 75, 1139 (1981); Surf. Sci. 158, 165 (1985).

${ }^{5}$ R. Ruppin, J. Chem. Phys. 76, 1681 (1982).

${ }^{6}$ H. Chew, J. Chem. Phys. 87, 1355 (1987).
${ }^{7}$ Y. S. Kim, P. T. Leung, and T. F. George, Surf. Sci. 195, 1 (1988).

${ }^{8}$ R. Carminati, J.-J. Greffet, C. Henkel, and J. M. Vigoureux, Opt. Commun. 261, 368 (2006); A. Moroz, ibid. 283, 2277 (2010).

${ }^{9}$ H. Mertens, A. F. Koenderink, and A. Polman, Phys. Rev. B 76, 115123 (2007).

${ }^{10}$ L. A. Blanco and F. J. Garcia de Abajo, Phys. Rev. B 69, 205414 (2004).

${ }^{11}$ A. Moroz, Ann. Phys. (NY) 315, 352 (2005). 
${ }^{12}$ W. L. Blacke and P. T. Leung, Phys. Rev. B 56, 12625 (1997);

R. M. Amos and W. L. Barnes, ibid. 59, 7708 (1999).

${ }^{13}$ P. T. Leung, Phys. Rev. B 42, 7622 (1990).

${ }^{14}$ J. Vielma and P. T. Leung, J. Chem. Phys. 126, 194704 (2007).

${ }^{15}$ W. Ekardt and Z. Penzar, Phys. Rev. B 34, 8444 (1986).

${ }^{16}$ O. Andreussi, S. Corni, B. Mennucci, and J. Tomasi, J. Chem. Phys. 121, 10190 (2004); M. Caricato, O. Andreussi, and S. Corni, J. Phys. Chem. B 110, 16652 (2006).

${ }^{17}$ V. G. Veselago, Sov. Phys. Usp. 10, 509 (1968).

${ }^{18}$ S. A. Ramakrishna, Rep. Prog. Phys. 68, 449 (2005); W. Cai and V. Shalaev, Optical Metamaterials: Fundamentals and Applications (Springer, New York, 2010).

${ }^{19}$ R. Ruppin and O. J. F. Martin, J. Chem. Phys. 121, 11358 (2004).

${ }^{20}$ J. Kastel and M. Fleischhauer, Phys. Rev. A 71, 011804 (2005).

${ }^{21}$ J. P. Xu, Y. P. Yang, H. Chen, and S. Y. Zhu, Phys. Rev. A 76, 063813 (2007).

${ }^{22}$ V. V. Klimov, Opt. Commun. 211, 183 (2002).

${ }^{23}$ V. V. Klimov and V. S. Letokhov, Laser Phys. 15, 61 (2005).

${ }^{24}$ H. T. Dung, S. Y. Buhmann, L. Knoll, D. G. Welsch, S. Scheel, and J. Kastel, Phys. Rev. A 68, 043816 (2003).

${ }^{25}$ H. Y. Xie, P. T. Leung, and D. P. Tsai, Solid State Commun. 149, 625 (2009).
${ }^{26}$ D. Guzatov and V. Klimov, arXiv:1203.5393 [physics, optics].

${ }^{27}$ E. Dulkeith, A. C. Morteani, T. Niedereichholz, T. A. Klar, J. Feldmann, S. A. Levi, F. C. J. M. van Veggel, D. N. Reinhoudt, M. Moller, and D. I. Gittins, Phys. Rev. Lett. 89, 203002 (2002).

${ }^{28}$ E. Dulkeith, M. Ringler, T. A. Klar, J. Feldmann, A. Munoz Javier, and W. J. Parak, Nano Lett. 5, 585 (2005).

${ }^{29}$ S. M. Barnett, B. Huttnerzk, R. Loudon, and R. Matloob, J. Phys. B: At. Mol. Opt. Phys. 293763 (1996).

${ }^{30}$ R. R. Chance, A. Prock, and R. Silbey, J. Chem. Phys. 62, 2245 (1975).

${ }^{31}$ H. Y. Chung, P. T. Leung, and D. P. Tsai, J. Chem. Phys. 136, 184106 (2012).

${ }^{32}$ D. R. Smith, W. J. Padilla, D. C. Vier, S. C. Nemat-Nasser, and S. Schultz, Phys. Rev. Lett. 84, 4184 (2000).

${ }^{33}$ See Ref. 24 for a discussion of the effect of the shifted frequency.

${ }^{34}$ Note that there exists different definitions for the relation between the field and the Green dyadic in the literature. Here we follow those in Ref. 3.

${ }^{35}$ See, e.g., Moroz's work in Ref. 8.

${ }^{36}$ R. Ruppin, Solid State Commun. 116, 411 (2000).

${ }^{37}$ E. Prodan, C. Radloff, N. J. Halas, and P. Nordlander, Science 302, 419 (2003) 